Article

\title{
Lung Fibrosis Is Improved by Extracellular Vesicles from IFN $\gamma$-Primed Mesenchymal Stromal Cells in Murine Systemic Sclerosis
}

\author{
Pauline Rozier ${ }^{1}$, Marie Maumus ${ }^{1}$, Alexandre Thibault Jacques Maria ${ }^{1,2}$, Karine Toupet ${ }^{1}$, Christian Jorgensen ${ }^{1,3}$, \\ Philippe Guilpain 1,2 (D) and Danièle Noël 1,3,*(D) \\ 1 IRMB, University of Montpellier, INSERM, 34295 Montpellier, France; pauline.rozier@inserm.fr (P.R.); \\ marie.maumus@inserm.fr (M.M.); a-maria@chu-montpellier.fr (A.T.J.M.); karine.toupet@inserm.fr (K.T.); \\ christian.jorgensen@inserm.fr (C.J.); p-guilpain@chu-montpellier.fr (P.G.) \\ 2 Department of Internal Medicine, Multi-Organic Diseases, CHU, 34295 Montpellier, France \\ 3 Clinical Immunology and Osteoarticular Disease Therapeutic Unit, Department of Rheumatology, CHU, \\ 34295 Montpellier, France \\ * Correspondence: daniele.noel@inserm.fr; Tel.: +33-4-67-33-04-73; Fax: +33-4-67-33-01-13
}

check for updates

Citation: Rozier, P.; Maumus, M.;

Maria, A.T.J.; Toupet, K.;

Jorgensen, C.; Guilpain, P.; Noël, D.

Lung Fibrosis Is Improved by

Extracellular Vesicles from

IFN $\gamma$-Primed Mesenchymal Stromal Cells in Murine Systemic Sclerosis.

Cells 2021, 10, 2727. https://doi.org/ $10.3390 /$ cells 10102727

Academic Editor: Nicoletta Del Papa

Received: 20 September 2021

Accepted: 7 October 2021

Published: 13 October 2021

Publisher's Note: MDPI stays neutral with regard to jurisdictional claims in published maps and institutional affiliations.

Copyright: (C) 2021 by the authors. Licensee MDPI, Basel, Switzerland. This article is an open access article distributed under the terms and conditions of the Creative Commons Attribution (CC BY) license (https:// creativecommons.org/licenses/by/ $4.0 /)$.

\begin{abstract}
Background: Systemic sclerosis (SSc) is a severe autoimmune disease for which mesenchymal stromal cells (MSCs)-based therapy was reported to reduce SSc-related symptoms in pre-clinical studies. Recently, extracellular vesicles released by MSCs (MSC-EVs) were shown to mediate most of their therapeutic effect. Here, we aimed at improving their efficacy by increasing the MSC-EV dose or by IFN $\gamma$-priming of MSCs. Methods: small size (ssEVs) and large size EVs (lsEVs) were recovered from murine MSCs that were pre-activated using 1 or $20 \mathrm{ng} / \mathrm{mL}$ of IFN $\gamma$. In the HOCl-induced model of SSc, mice were treated with EVs at day 21 and sacrificed at day 42. Lung and skin samples were collected for histological and molecular analyses. Results: increasing the dose of MSC-EVs did not add benefit to the dose previously reported to be efficient in SSc. By contrast, IFN $\gamma$ pre-activation improved MSC-EVs-based treatment, essentially in the lungs. Low doses of IFN $\gamma$ decreased the expression of fibrotic markers, while high doses improved remodeling and anti-inflammatory markers. IFN $\gamma$ pre-activation upregulated iNos, IL1ra and Il6 in MSCs and ssEVs and the PGE2 protein in lsEVs. Conclusion: IFN $\gamma$-pre-activation improved the therapeutic effect of MSC-EVs preferentially in the lungs of SSc mice by modulating anti-inflammatory and anti-fibrotic markers.
\end{abstract}

Keywords: mesenchymal stromal cell; scleroderma; extracellular vesicles; exosomes; microvesicles; therapy

\section{Introduction}

Systemic sclerosis (SSc) is an autoimmune disease with a severe prognosis due to generalized fibrosis and vasculopathy [1]. Currently, symptomatic management is the only strategy available to relieve patients and no curative treatment can reverse the disease. For some patients, immunosuppressive drugs and hematopoietic stem cell transplantation could be effective in stopping the course of the disease, but these also come with severe side effects [2]. More recently, mesenchymal stromal cells (MSCs) have demonstrated therapeutic benefit in preclinical models of SSc thanks to their pleiotropic properties. Currently, MSCs are being evaluated in the clinics, and promising results have been reported [3].

MSCs exert their therapeutic function through the release of many soluble mediators that are secreted in the extracellular milieu and/or within extracellular vesicles (EVs). EVs are a heterogeneous population of vesicles that are characterized by their size and biogenesis [4]. Apart from apoptotic bodies that are released by apoptotic cells, the two main EV subtypes are exosomes, which are below $150 \mathrm{~nm}$ in diameter and generated via the endosomal pathway inside multivesicular bodies, and microvesicles, or microparticles, 
whose size is above $120 \mathrm{~nm}$ and that form by budding of the plasma membrane. Because of overlapping sizes, current isolation procedures allow for the isolation of small size EVs (ssEVs) and large size EVs (lsEVs), which are enriched in exosomes and microvesicles, respectively [5]. Both types of EVs contain a cargo of proteins, lipids, and nucleic acids (including DNA, mRNA, miRNA) that mediate the functions of parental cells.

Using the HOCl-induced murine model of SSc, we previously reported that murine and human MSCs from bone marrow (BM) and adipose tissue (AT) are efficient to stop the course of the disease and prevent skin and lung sclerosis [6,7]. Recently, we demonstrated that EVs isolated from murine BM-MSCs and human AT-MSCs reproduce the therapeutic effect of parental cells [8]. We showed that both ssEVs and lsEVs powerfully stop disease progression and regulate expression of fibrotic and remodeling markers in HOCL-induced murine SSc. They also down-regulate inflammation in the skin and lungs of treated mice. The beneficial effect has been associated with the presence of miR-29a-3p in murine ssEVs and lsEVs as well as in total EVs from human AT-MSCs. MiR-29a-3p was shown to act via the regulation of type I and III collagens, apoptotic factors (Bax, Bcl2, Bcl-xl), methylation-regulating genes (Tet1, Dnmt3a) and Pdgfrbb in the skin of SSc mice.

In the present study, we investigated the possibility of enhancing the therapeutic effect of ssEVs and lsEVs isolated from murine BM-MSCs (mMSC) in the murine model of SSc. We evaluated the interest of using a higher dose of EVs or the pre-activation of MSCs by IFN $\gamma$, which is known to stimulate their immunosuppressive effect, before EV isolation [9-11]. We also tested whether IFN $\gamma$ pre-treatment of MSCs could up-regulate the production of immunosuppressive factors and their release within EVs, thereby enhancing their therapeutic effect in SSc.

\section{Materials and Methods}

\subsection{Mesenchymal Stromal Cell Expansion}

C57BL/6 mice-derived mMSCs were cultured in proliferative medium containing DMEM, $10 \%$ fetal calf serum (FCS), $100 \mu \mathrm{g} / \mathrm{mL}$ penicillin/streptomycin and $2 \mathrm{mmol} / \mathrm{mL}$ glutamine. Their characterization by phenotyping and tri-lineage differentiation potential has been reported before [12]. We used mMSCs between passage 12 and 18 for the following experiments.

\subsection{Production and Isolation of EVs}

EV subtypes were produced from mMSCs and characterized according to ISEV recommendations as previously described $[8,13]$. Briefly, mMSCs were seeded at 7000 cells $/ \mathrm{cm}^{2}$ in proliferative medium for $24 \mathrm{~h}$. After a wash with phosphate buffer saline (PBS), the production medium (DMEM containing 3\% EV-free FCS) was added for $48 \mathrm{~h}$. When necessary, recombinant mouse IFN $\gamma(1$ or $20 \mathrm{ng} / \mathrm{mL}$ ) was added (R\&D Systems, Bio-Techne, Noyal Chatillon, France). After removing cells and debris thanks to low speed centrifugation, lsEVs were pelleted by a first ultracentrifugation at $18000 \times \mathrm{g}, 4^{\circ} \mathrm{C}$, for $1 \mathrm{~h}$ and ssEVs were pelleted by a second ultracentrifugation at $100,000 \times g, 4^{\circ} \mathrm{C}$, for $2 \mathrm{~h}$. Both pellets were then washed in PBS and submitted to another ultracentrifugation round at $100,000 \times g$ for $2 \mathrm{~h}$. EV subtypes were characterized according to their morphology and their size thanks to cryoTEM and nanotracking analysis. Protein content was evaluated thanks to cytometry and western blot experiments, as reported elsewhere [8].

\subsection{Animal Model and Histopathological Analysis}

Mice were raised in the conditions required by the European guidelines for the care and use of laboratory animals (2010/63/UE). This project was approved by the Regional Ethics Committee on Animal Experimentation (APAFIS\#53512016050919079187). SSc was induced onto six-week-old female BALB/cJRj mice (Janvier Labs, Le Genest-Saint-Isle, France) using daily $\mathrm{HOCl}$ intradermic injections for 42 days $(150 \mu \mathrm{L}$ into two sites at the base of the tail). Once a week, skin thickness was measured thanks to a caliper. At day 21, groups of 7-8 mice were formed to homogenize the mean skin thickness between groups. 
Mice received one intravenous injection $(100 \mu \mathrm{L})$ of either $\mathrm{NaCl} 0.9 \%$ (control group), 250,000 mMSCs or $250 \mathrm{ng}$ EVs (or $1500 \mathrm{ng}$ depending on the experiment). Mice were split in the cages to minimize bias between cages. At day 42, blood, lungs and skin biopsies ( $6 \mathrm{~mm}$ punchs) were recovered. Blood was allowed to coagulate at room temperature (RT) at least $30 \mathrm{~min}$ and was then subjected to centrifugation at $1000 \times g$, RT for $10 \mathrm{~min}$ to recover the serum, which was stored at $-80^{\circ} \mathrm{C}$. Skin or lung samples for molecular analysis were stored at $-80^{\circ} \mathrm{C}$. Samples dedicated to histology were directly fixed in $4 \%$ formaldehyde before paraffin embedding and routine histology. Histological slides ( $5 \mu \mathrm{m}$ thick) were stained by Masson's trichrome staining and analyzed with Nanozoomer (Hamamatsu) and NDP.view2 software to measure dermal thickness.

\subsection{RNA Extraction and RT-qPCR}

Skin and lung samples were grinded in RLT-buffer containing $1 \% \beta$-mercaptoethanol and processed using the RNeasy Mini Kit for total RNA extraction, according to manufacturer's recommendations (Qiagen, Courtaboeuf, France). RNA (500 ng) was reverse transcribed by M-MLV reverse transcriptase (ThermoFisher Scientific, Illkirch-Graffenstaden, France). Using specific primers, main fibrotic (Col1a1, Col3a1, Tgf $\beta 1$, Tgf $\beta R 2)$, remodeling (MMP9, MMP1/TIMP1), inflammatory (IL1 $\beta, T N F \alpha)$ and oxidative stress (Cox2, Hmox1, Sod2) markers were analysed (Table 1). Real time PCR was performed on 20 ng cDNA using the Master SYBR Green I mix (Roche Diagnostics, Meylan, France). TATA binding protein $(T b p)$ was used as the housekeeping gene and data were expressed as Tbp relative expression and as fold change compared to control non-treated group using the formulae $2^{-\Delta \mathrm{Ct}}$ or $2^{-\Delta \Delta \mathrm{Ct}}$, respectively.

Table 1. List of primers for gene analysis by RT-qPCR.

\begin{tabular}{|c|c|c|}
\hline Gene Name & Sequence Forward & Sequence Reverse \\
\hline $\operatorname{Acta2}(\alpha S m a)$ & AAGGCCAACCGGGAGAAAAT & AGCCAAGTCCAGACGCATGA \\
\hline Col1a1 & TGTTCAGCTTTGTGGACCTC & TCAAGCATACCTCGGGTTTC \\
\hline Col3a1 & CGGTGAACGGGGCGAAGCTGGTT & GACCCCTTTCTCCTGCGGCTCCT \\
\hline $\operatorname{Cox} 2$ & GCATTCTTTGCCCAGCACTT & AGACCAGGCACCAGACCAAAGA \\
\hline Gapdh & GGTGCTGAGTATGTCGTGGA & GTGGTTCACACCCATCACAA \\
\hline$H g f$ & TGCCCTATTTCCCGTTGTGA & CGCTTCTCCTCGCСTCTCTC \\
\hline Hmox1 & GCAGAGCCGTCTCGAGCATA & GCATTCTCGGCTTGGATGTG \\
\hline Il1ra & AGGCCCCACCACCAGCTTTGA & GGGGCTCTTCCGGTGTGTTGGT \\
\hline$I l 1 \beta$ & TTTGACAGTGATGAGAATGACCTGTTC & TCATCAGGACAGCCCAGGTCAAAG \\
\hline Il6 & TGGGACTGATGCTGGTGACA & TTCCACGATTTCCCAGAGAACA \\
\hline$i$ Nos & CCTTGTTCAGCTACGCCTTC & GCTTGTCACCACCAGCAGTA \\
\hline Mmp1 & TTCAAAGGCAGCAAAGTATGGGCT & CCAGTCTCTTCTTCACAAACAGCAGCA \\
\hline Mmp9 & TCCAGTTTGGTGTCGCGGAGCACG & CAGGGGGAAAGGCGTGTGCCAGA \\
\hline Nfe2l2 & CGCCAGCTACTCCCAGGTTG & ACTTTCAGCGTGGCTGGGGA \\
\hline Sod2 & TCAGGACCCATTGCAAGGAA & TGTGGCCGTGAGTGACGTTT \\
\hline$T b p$ & GGGAGAATCATGGACCAGAA & CCGTAAGGCATCATTGGACT \\
\hline $\operatorname{Tgf} \beta 1$ & TGCGCTTGCAGAGATTAAAA & CTGCCGTACAACTCCAGTGA \\
\hline$T g f \beta R 2$ & CGACCCCAAGCTCACCTACC & CAACAACAGGTCGGGACTGC \\
\hline Timp1 & CTCCGCCCTTCGCATGGACATT & GGGGGCCATCATGGTATCTGCTCT \\
\hline $\operatorname{Tnf\alpha }$ & AGCCCACGTCGTAGCAAACCA & TGTCTTTGAGATCCATGCCGTTGGC \\
\hline
\end{tabular}


MSC and EV samples were treated with Qiazol reagent (ThermoFisher Scientific) using miRNeasy Mini or Micro Kit, respectively (Qiagen, Courtaboeuf). Reverse transcription was performed using M-MLV Reverse Transcriptase kit (ThermoFisher Scientific), followed by real-time PCR on $10 \mathrm{ng}$ cDNA using specific primers (Table 1) and SYBR Green I Master mix (Roche Diagnostics). Data were normalized to the expression of D-glyceraldehyde-3phosphate dehydrogenase (Gapdh) housekeeping gene for mMSCs and expressed using the formulae $2^{-\Delta \mathrm{CT}}$. For miRNA, reverse transcription of $10 \mathrm{ng}$ total RNA was performed according to the recommendations of TaqMan ${ }^{\circledR}$ MicroRNA Reverse Transcription Kit (ThermoFisher Scientific). Real-time PCR was performed on 2 ng cDNA using Taqman microRNA hsa-miR29a-3p (Life Technologies, Villebon-sur-Yvette, France) and Taqman Master Mix II no UNG (Life Technologies). Data were normalized to the expression of U6 snRNA (Life Technologies) for mMSCs and data were expressed as 100/Ct for EVs.

\subsubsection{Measure of Advanced Oxidation Protein Products}

Sera or a range of $200 \mu \mathrm{L}$ of standard chloramine T solution (0 to $1000 \mu \mathrm{M})$ were incubated with $20 \mu \mathrm{L}$ acetic acid and $10 \mu \mathrm{L}$ potassium iodide $(1.16 \mathrm{M})$ at RT. Optical densities were measured at $340 \mathrm{~nm}$ on a microplate reader (Varioskan, ThermoFisher Scientific), before incubation and each minute for $10 \mathrm{~min}$. AOPP concentrations were expressed as chloramine T equivalents $(\mu \mathrm{M})$.

\subsubsection{Measure of Anti-Oxidant Capacity}

Anti-oxidant capacity (AOC) was measured on sera diluted to $1 / 10$ or a standard range of Trolox using the Antioxidant Assay Kit (Cayman Chemical, Interchim, Montluçon, France). The absorbance was read at $750 \mathrm{~nm}$ and AOC was expressed as $\mathrm{mM}$ Trolox equivalents.

\subsection{ELISA}

After the last step of centrifugation, EVs were suspended in $100 \mu \mathrm{L}$ of NP40 cell lysis buffer (Invitrogen, ThermoFisher Scientific). Total proteins were quantified using MicroBCA assay kit (ThermoFisher Scientific). Samples were stored at $-20{ }^{\circ} \mathrm{C}$ until quantification using DuoSet ${ }^{\circledR}$ ELISA kits for HGF, IL1RA, IL6, TGF $\beta 1$ (R\&D Systems) and prostaglandin E2 (PGE2) using Multi-format ELISA kit (Arbor Assays, Clinisciences, Nanterre) on $1 \mu \mathrm{g}$ of total proteins.

\subsection{Analysis of miRNA Profiles}

The miRNA profile of EVs was analysed by HTG Next-Generation Sequencing using an Illumina NextSeq 500 system by the company Firalis (Huningue, France). We selected the 277 miRNAs that were common to human and mouse species and normalized the cpm value of each miRNA on the total cpm count. We then selected the 131 miRNAs that had a cpm value $>500$.

Expression of miRNAs in IFN $\gamma$-pre-activated ssEVs and lsEVs were normalized to their expression in non-activated ssEVs and lsEVs. We selected the miRNAs that were upand down-regulated (FC $>1.5$ and FC $<-1.5$, respectively) in IFN $\gamma$-pre-activated ssEVs and lsEVs.

\subsection{Gene Ontology Pathway Analysis}

The identification of validated target genes modulated by miRNAs in naive or IFN $\gamma$ pretreated lsEVs and ssEVs were done using TaRbase [14]. Enrichr and Panther software were used to perform biological pathway enrichment analysis and Gene Ontology (GO) enrichment analysis for identification of gene enrichment in cellular component and molecular function [15-17].

\subsection{Statistical Analysis}

Statistical analyses were performed using GraphPad 8 Prism Software. Data distribution was evaluated using the Shapiro-Wilk normality test. Each single group was compared 
to the control group using the Student t-test or the Mann-Whitney test when values were parametric or non-parametric, respectively. For values normalized to 1 , a one sample t-test or Wilcoxon test were performed when values assumed or not a normal distribution, respectively. Data are presented as mean \pm SEM.

\section{Results}

\subsection{A High Dose of MSC-EVs Was Not Beneficial to SSc Mice}

Isolation and characterization of ssEVs and lsEVs from murine BM-MSCs have been described previously [8]. After systemic injection of these MSC-derived ssEVs and lsEVs, we showed that the two sub-populations of EVs can reduce clinical symptoms and histopathological alterations in SSc. With the aim to improve treatment efficacy, we tested whether a higher dose of lsEVs might be beneficial. By comparison with the dose of $250 \mathrm{ng}$ used in the previous study, a high dose of lsEVs (1500 ng) did not stop disease progression as shown by a continuous skin thickness increase, which was similar to non-treated mice, and a higher skin thickness measured at day 42 (Figure 1A,B).

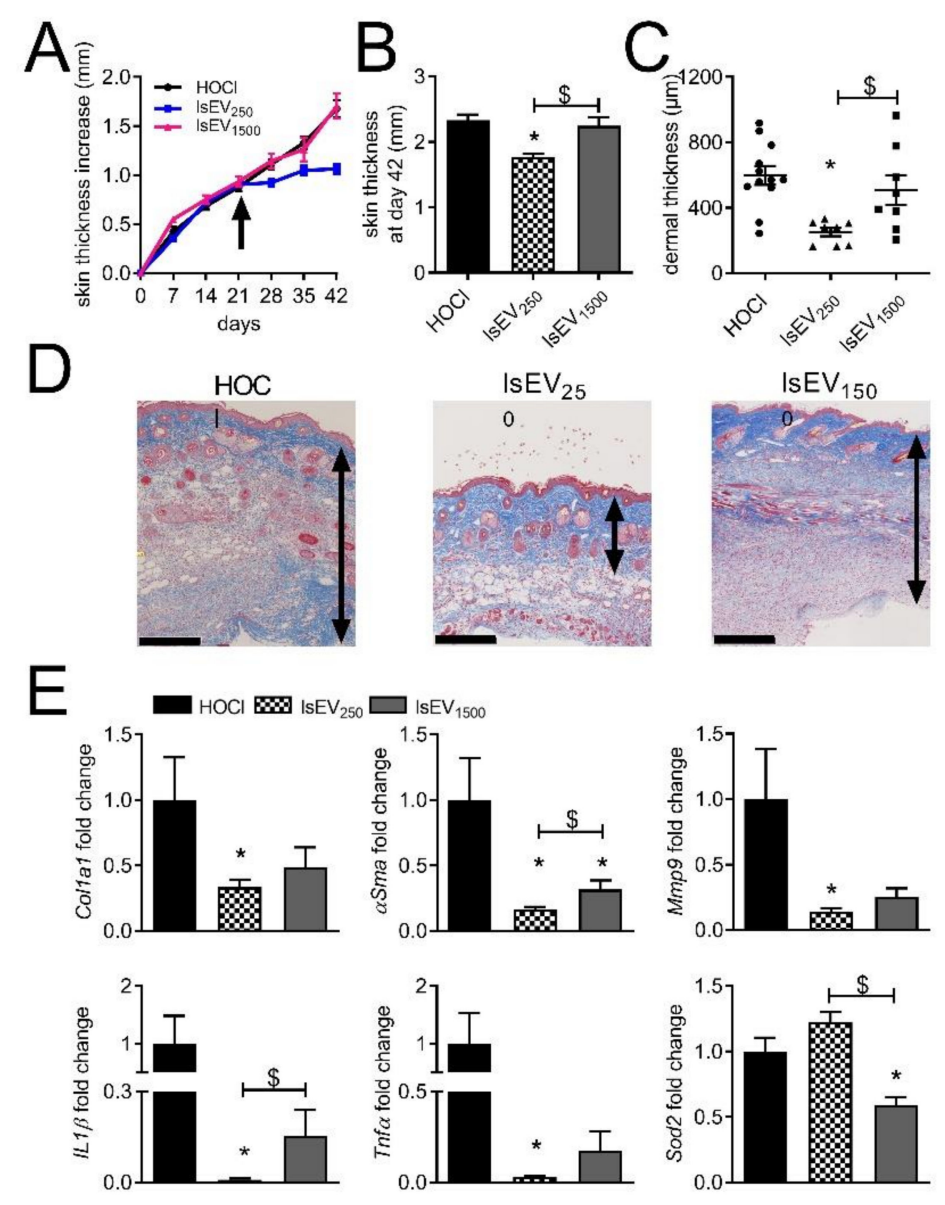

Figure 1. Dose effect of lsEVs isolated from MSCs in the murine model of HOCl-induced SSc. (A) Measures of the skin thickness increase in control mice $(\mathrm{HOCl})$ and mice that have been injected with 250 or $1500 \mathrm{ng}$ of large size extracellular vesicles ( $\mathrm{lsEV}_{250}$ or $\mathrm{lsEV}_{1500}$, respectively) on day 21 (arrow). (B) Mean skin thickness in the different groups of mice at day 42. (C) Mean dermal thickness on histological sections of skin from the three groups of mice. (D) Photographs of representative histological sections of skin after Masson's trichrome staining (the double arrow delineates the dermis; scale bar, $250 \mu \mathrm{m}$ ). (E) Gene expression in skin samples as expressed as fold change in treated versus $\mathrm{HOCl}$ control mice. Data are presented as mean $\pm \mathrm{SEM}(n=8$ to 12 per group; * $p<0.05$ versus control or $\$: p<0.05$ versus the indicated group of mice). 
On histological sections, the dermal thickness in mice receiving the high dose of lsEVs was similar to non-treated mice and higher than in mice receiving the low dose of lsEVs (Figure 1C,D). Contrary to the low dose, the high dose of lsEVs did not significantly improve the expression of several fibrotic (Col1a1), remodeling (Mmp9) and inflammatory $(I l 1 \beta, T n f \alpha)$ markers (Figure 1E). Expression of $\alpha S m a$ and Il1 $\beta$ was higher in mice treated with the high dose versus the low dose of lsEVs while the anti-oxidant marker Sod 2 was lower. Similar results were observed in the lungs. On histological sections, no improvement in pulmonary fibrosis was noticed as indicated by a dense and infiltrated parenchyma in mice injected with the high dose of lsEVs (Supplementary Figure S1A). Expression of fibrotic markers (Col1a1, Col3a1, $\alpha$ Sma), did not improve although the expression of Mmp9 and Il1 $\beta$ was reduced to similar levels as the low dose of lsEVs (Supplementary Figure S1B). Of note, the mRNA levels of Mmp1 (as illustrated by the ratio Mmp1/Timp1), Hmox1, Nfe2l2 and Sod2 increased after the injection of the high dose of lsEVs. Altogether, the results indicated that the high dose of lsEVs did not improve the clinical and histological features of SSc, even though remodeling and anti-oxidative capacity improved in the lungs.

\subsection{Low Dose IFN $\gamma$ Pre-Activation Improved the Anti-Fibrotic Effect of MSC-EVs in Lungs}

We then evaluated whether the pre-activation of MSCs by a low dose of IFN $\gamma(1 \mathrm{ng} / \mathrm{mL})$ could improve the therapeutic effect of ssEVs and lsEVs, notably their anti-inflammatory effect [18]. Although disease progression was stopped or slowed down in all treated groups, pre-activated EV subtypes (ssEV A1 and lsEV A1) were less efficient than non-activated EV subtypes (ssEV NA and lsEV NA) (Figure 2A). At day 42, the skin thickness of mice that received ssEV A1 and lsEV A1 was significantly thicker as compared to non-activated EVs (Figure 2B). The measure of dermal thickness on skin histological sections indicated a lower thickness in all groups and revealed a heterogeneity of response in the ssEV A1 group (Figure 2C,D). All the fibrotic and inflammatory markers were significantly lower in the treated groups, even though lsEV A1 were less efficient than lsEV NA to reduce the expression of Tgfß1, $\alpha S m a$ and Tnfa (Figure 2E). As expected, the gelatinase Mmp9 was reduced in all treated groups and the Mmp1/Timp1 ratio, which is representative of matrix remodeling, was increased. We also measured the advanced oxidized protein products (AOPP) concentration in the sera as a marker of oxidative stress. Interestingly, AOPP levels were significantly lower in all treated groups (Figure 2F).

Again, lsEV A1 were less efficient than lsEV NA to decrease AOPP levels. The antioxidative capacity (AOC) in the sera of treated groups was not increased as compared to that of the control group and was even decreased in the ssEV A1 group (Figure 2F). As a result, the AOPP/AOC ratio was significantly reduced in all treated groups, indicating an anti-oxidative effect of EVs, but to a lesser extend in the lsEV A1 group.

In the lungs, the analysis of histological sections showed fewer collagen deposits in treated groups, regardless of EV subtype (Figure 3A).

At the molecular level, both ssEV A1 and lsEV A1 decreased the expression of fibrotic markers (Col3a1, Tgf $\beta 1, T g f \beta r 2$ ) as compared to ssEV NA and lsEV NA or control group (Figure 3B). Nevertheless, ssEV A1 and lsEV A1 did not reduce the expression of Mmp 9 and the Mmp1/Timp1 ratio was unchanged. The inflammatory markers Il1 $\beta$ and Tnf $\alpha$ were lower in all treated groups. Overall, MSC pre-activation did not improve the efficacy of the two EV subtypes on cutaneous fibrosis but improved at least partially lung fibrosis by reducing the expression of all the fibrotic and inflammatory markers.

\subsection{High Dose IFN $\gamma$ Pre-Activation Improved Remodeling and Anti-Inflammatory Effect of MSC-EVs in Lungs}

With the hope to increase these anti-fibrotic and anti-inflammatory effects of MSC-EVs in the lungs and possibly in the skin, we tested a higher dose of IFN- $\gamma(20 \mathrm{ng} / \mathrm{mL})$ to pre-activate MSCs. Both pre-activated MSC-EV subtypes (ssEV A20 and lsEV A20) stopped the disease course and reduced the skin thickness to similar levels as non-activated EV subtypes, but lsEV A20 were more efficient than ssEV A20 (Figure 4A,B). Histological sections of skin revealed a lower dermal thickness in all treated groups (Figure 4C). Most 
of the molecular markers improved in the skin of treated groups except in the ssEV A20 group for Col1 $\alpha 1, T g f \beta r 2$, Mmp1/Timp1, Il1 $\beta$, Tnf $\alpha$ (Figure 4D). Altogether, independently on pre-activation, lsEVs seemed more efficient than ssEVs, in particular on remodeling and inflammatory markers in the skin.

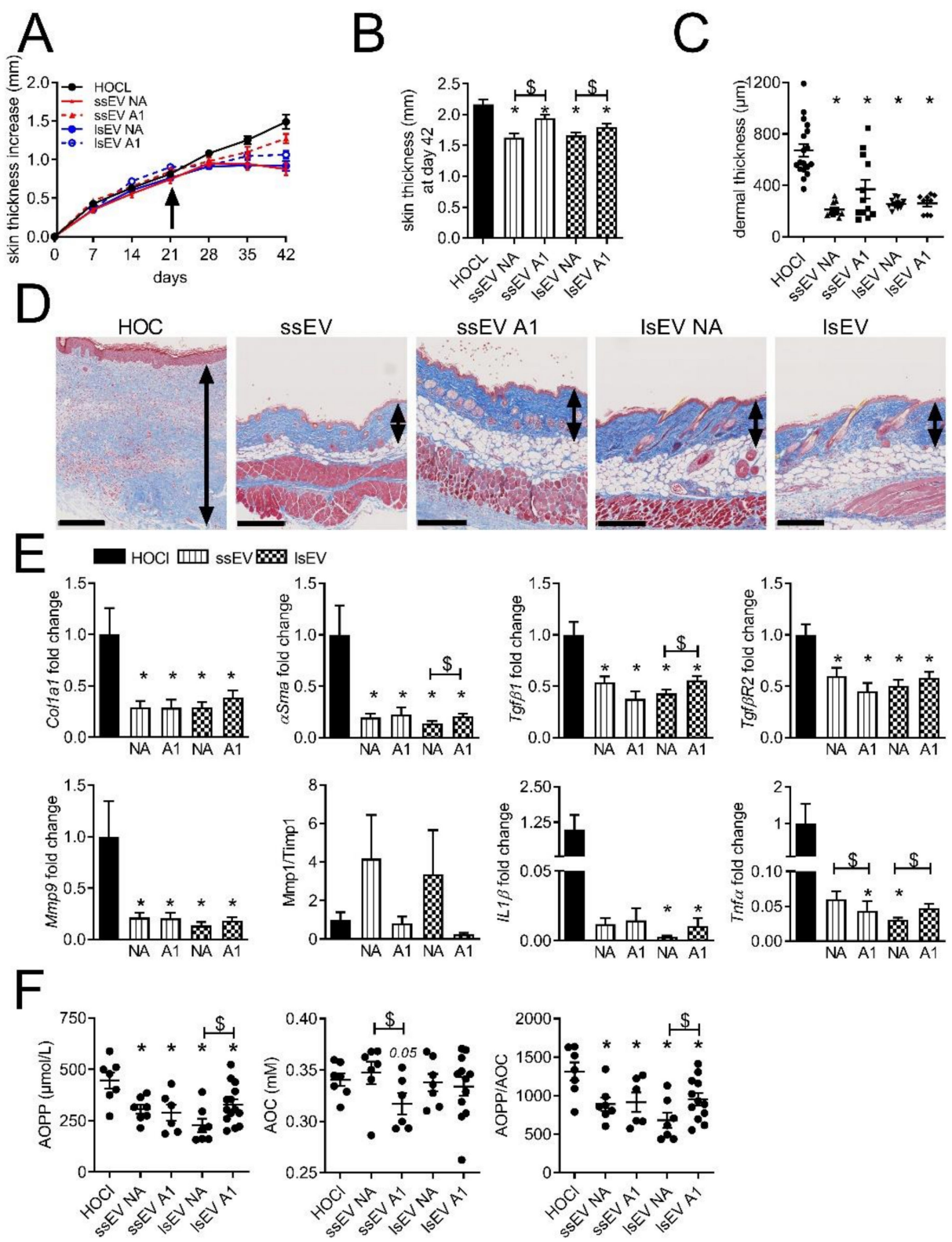

Figure 2. Therapeutic effect of EVs isolated from MSCs pre-activated with low dose of IFN $\gamma$ in skin from HOCl-induced SSc. (A) Measures of the skin thickness increase in control mice (HOCl) and mice that have been injected with small size or large size extracellular vesicles isolated from non-activated MSCs (ssEV NA or lsEV NA) or MSCs pre-activated by $1 \mathrm{ng} / \mathrm{mL}$ IFN $\gamma$ (ssEV A1 or lsEV A1) on day 21 (arrow). (B) Mean skin thickness in the different groups of mice at day 42. (C) Mean dermal thickness on histological sections of skin from the different groups of mice. (D) Photographs of representative histological sections of skin after Masson's trichrome staining (the double arrow delineates the dermis; scale bar, $250 \mu \mathrm{m}$ ). (E) Gene expression in skin samples as expressed as fold change in treated versus $\mathrm{HOCl}$ control mice. (F) Quantification of advanced oxidation protein products (AOPP) and of anti-oxidant capacity (AOC) in the serum of mice as expressed as chloramine $\mathrm{T}$ and Trolox equivalents, respectively, and expression of the AOPP/AOC ratio. Data are presented as mean $\pm \operatorname{SEM}\left(n=12\right.$ to 18 per group from 2 separate experiments (except for data in $\mathrm{f}$ ); ${ }^{*} p<0.05$ versus control or $\$: p<0.05$ versus the indicated group of mice). 


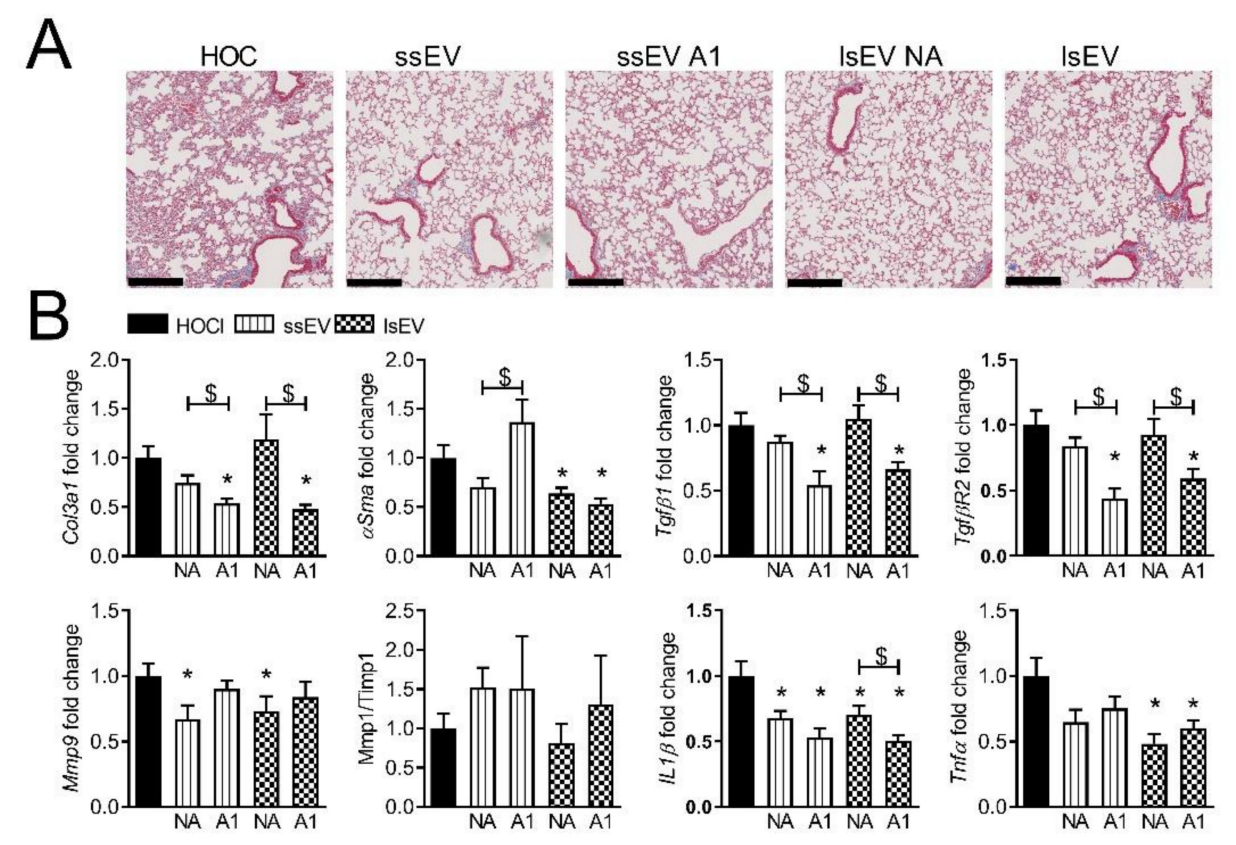

Figure 3. Therapeutic effect of EVs isolated from MSCs pre-activated with low dose of IFN $\gamma$ in lungs from HOCl-induced SSc. (A) Photographs of representative histological sections of lungs after Masson's trichrome staining in control mice $(\mathrm{HOCl})$ and mice that have been injected with small size or large size extracellular vesicles isolated from non-activated MSCs (ssEV NA or lsEV NA) or MSCs pre-activated by $1 \mathrm{ng} / \mathrm{mL}$ IFN $\gamma$ (ssEV A1 or lsEV A1) (scale bar, $250 \mu \mathrm{m}$ ). (B) Gene expression in lung samples as expressed as fold change in treated versus $\mathrm{HOCl}$ control mice. Data are presented as mean $\pm \operatorname{SEM}(n=12$ to 18 per group from two separate experiments (except for data in $\mathrm{f}$ ); $: p<0.05$ versus control or $\$: p<0.05$ versus the indicated group of mice).

In the lungs, improvement of several fibrotic, remodeling and inflammatory markers was observed in all treated groups even though statistical significance was not reached for some markers in this specific experiment (Figure 4E). The pre-activated ssEVs A20 and lsEVs A20 seemed to be more potent than non-activated EVs to improve $\alpha S m a, T g f \beta r 2$ as well as all remodeling and inflammatory mediators (Figure 4E). No further beneficial effect was observed with lsEV A20 as compared to ssEV A20. Overall, EVs isolated from MSCs pre-activated with a high dose of IFN $\gamma$ tended to improve all molecular markers in the lungs.

\subsection{IFN $\gamma$ Pre-Activation Up-Regulated Anti-Inflammatory Factors in MSCs and MSC-EVs}

To better understand the mechanism of action of MSC-EVs, we compared the miRNA profile of EVs isolated from non-activated MSCs and MSCs pre-treated with a high dose of IFN $\gamma$ (Table 2).

We selected the miRNAs that were up- and down-regulated by a fold change $>1.5$ and found that most of the modulated miRNAs were down-regulated by IFN $\gamma$ (Figure 5A). Surprisingly, no miRNA was down-regulated in both ssEVs and lsEVs. We then performed a functional enrichment analysis on experimentally validated target genes. Using miRTarBase, we identified 27,979 target genes that were analyzed for biological pathway enrichment using Enrichr software. The first overrepresented biological pathway was related to IFN $\gamma$ (Figure 5B). Target genes were also classified into distinct functional categories, including molecular functions and cellular components, using GO Term Enrichment Analysis (Figure 5C). 


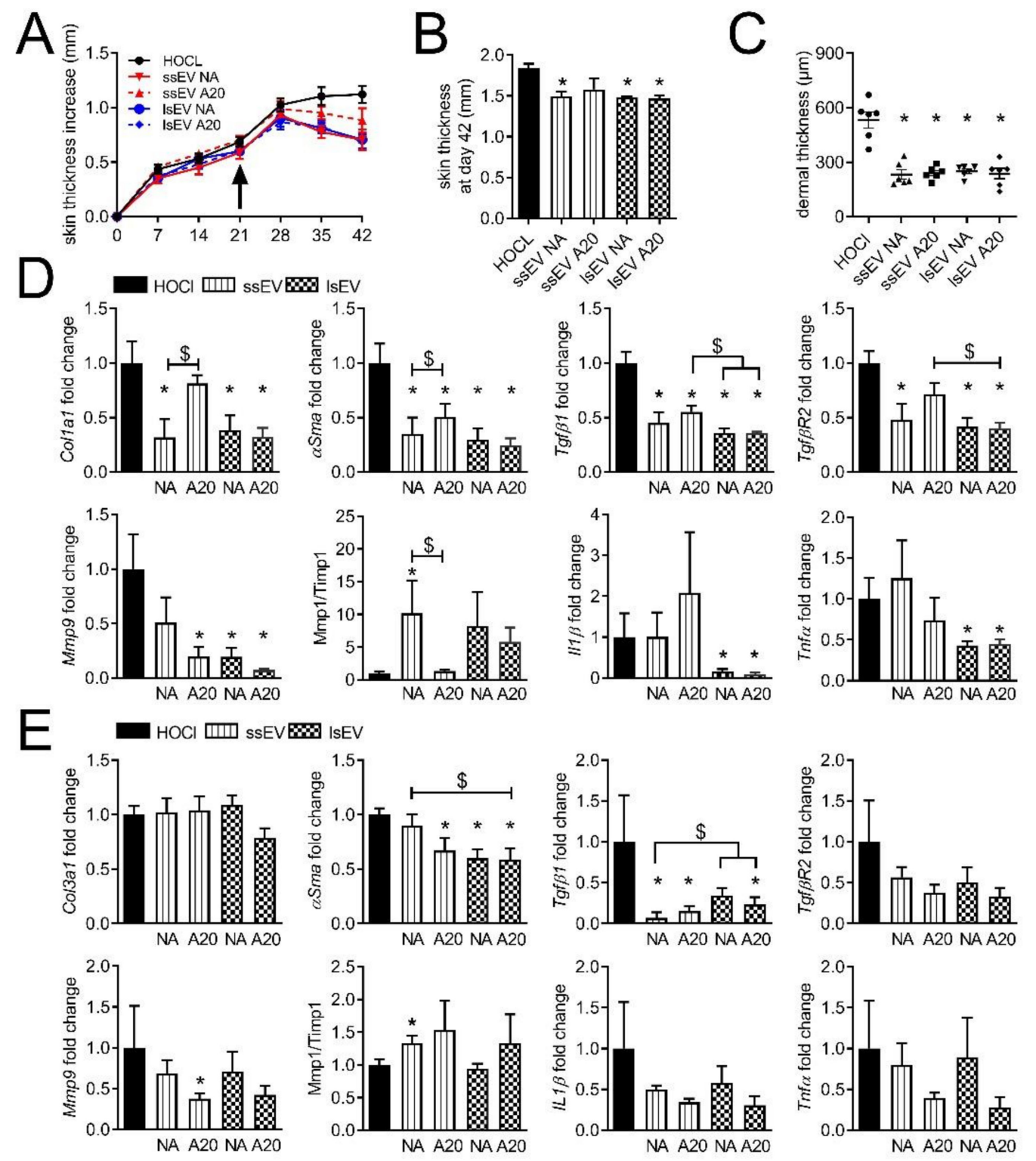

Figure 4. Therapeutic effect of EVs isolated from MSCs pre-activated with high doses of IFN $\gamma$ in the murine model of HOCl-induced SSc. (A) Measures of the skin thickness increase in control mice $(\mathrm{HOCl})$ and mice that have been injected with small size or large size extracellular vesicles isolated from non-activated MSCs (ssEV or lsEV NA) or MSCs pre-activated by $20 \mathrm{ng} / \mathrm{mL} \mathrm{IFN \gamma}$ (ssEVs or lsEVs A20) on day 21 (arrow). (B) Mean skin thickness in the different groups of mice at day 42. (C) Mean dermal thickness on histological sections of skin from the different groups of mice. (D) Gene expression in skin samples as expressed as fold change in treated versus $\mathrm{HOCl}$ control mice. (E) Gene expression in lung samples as expressed as fold change in treated versus $\mathrm{HOCl}$ control mice. Data are presented as mean $\pm \operatorname{SEM}\left(n=6\right.$ per group; ${ }^{*}: p<0.05$ versus control or $\$: p<0.05$ versus the indicated group of mice). 
Table 2. List of modulated miRNAs.

\begin{tabular}{|c|c|c|c|c|c|c|}
\hline & \multicolumn{2}{|c|}{ MSC } & \multicolumn{2}{|c|}{ ssEV } & \multicolumn{2}{|c|}{ lsEV } \\
\hline & $-\mathrm{IFN} \gamma$ & $+\mathrm{IFN} \gamma$ & $-\mathrm{IFN} \gamma$ & $+\mathrm{IFN} \gamma$ & $-\mathrm{IFN} \gamma$ & $+\mathrm{IFN} \gamma$ \\
\hline let-7a-5p & 39,871 & 33,617 & 10,128 & 5963 & 21,047 & 21,213 \\
\hline let-7b-5p & 47,447 & 35,255 & 8648 & 4556 & 21,621 & 21,476 \\
\hline let-7c-3p & 403 & 0 & 0 & 0 & 0 & 0 \\
\hline let-7c-5p & 57,948 & 42,747 & 11,281 & 6154 & 26,131 & 26,347 \\
\hline let-7d-3p & 1097 & 879 & 1585 & 1000 & 1544 & 1455 \\
\hline let-7d-5p & 17,079 & 16,250 & 5002 & 3356 & 9475 & 8869 \\
\hline let-7e-3p & 253 & 0 & 0 & 0 & 0 & 0 \\
\hline let-7e-5p & 7938 & 6261 & 1621 & 1023 & 4224 & 4091 \\
\hline let-7f-1-3p & 245 & 114 & 0 & 0 & 0 & 0 \\
\hline let-7f-5p & 21,504 & 21,024 & 8136 & 4975 & 15,443 & 15,217 \\
\hline let-7g-5p & 6287 & 7170 & 3143 & 2408 & 4563 & 4024 \\
\hline let-7i-5p & 33,329 & 30,098 & 8184 & 4723 & 16,303 & 13,232 \\
\hline miR-100-5p & 7165 & 7546 & 2619 & 1675 & 5070 & 4458 \\
\hline miR-101-3p & 513 & 1572 & 1757 & 2085 & 1188 & 1221 \\
\hline miR-103a-2-5p & 367 & 168 & 0 & 0 & 0 & 0 \\
\hline miR-103a-3p & 2655 & 2334 & 1695 & 1429 & 1978 & 1613 \\
\hline miR-106b-3p & 521 & 275 & 0 & 631 & 0 & 0 \\
\hline miR-106b-5p & 1881 & 2134 & 3213 & 3287 & 2228 & 2239 \\
\hline miR-107 & 1667 & 1684 & 1127 & 1070 & 1170 & 0 \\
\hline miR-1247-5p & 2752 & 1116 & 0 & 598 & 1544 & 1207 \\
\hline miR-1249 & 1213 & 626 & 4544 & 4571 & 3003 & 2429 \\
\hline miR-125a-3p & 745 & 328 & 0 & 0 & 0 & 0 \\
\hline miR-125a-5p & 13,783 & 15,755 & 5304 & 3068 & 11,867 & 10,808 \\
\hline miR-125b-1-3p & 3316 & 944 & 0 & 0 & 0 & 0 \\
\hline miR-125b-5p & 55,996 & 50,774 & 15,833 & 8527 & 31,962 & 31,144 \\
\hline miR-126-3p & 0 & 0 & 4183 & 4440 & 1692 & 1555 \\
\hline miR-126-5p & 0 & 0 & 2105 & 2089 & 1070 & 0 \\
\hline miR-128-3p & 454 & 424 & 0 & 668 & 0 & 0 \\
\hline miR-130a-3p & 7587 & 9334 & 3461 & 1860 & 5813 & 5799 \\
\hline miR-130b-3p & 1339 & 2413 & 1193 & 821 & 2200 & 2280 \\
\hline miR-140-5p & 1309 & 1419 & 0 & 0 & 1333 & 0 \\
\hline miR-142-5p & 0 & 0 & 5341 & 5417 & 2163 & 1799 \\
\hline miR-143-3p & 27,877 & 31,702 & 14,591 & 8415 & 25,698 & 21,680 \\
\hline miR-144-3p & 0 & 0 & 12,449 & 18,149 & 3434 & 2808 \\
\hline miR-145-5p & 37,004 & 33,556 & 16,311 & 8731 & 36,226 & 35,290 \\
\hline miR-146b-5p & 4779 & 7806 & 1639 & 1519 & 3429 & 4867 \\
\hline miR-148a-3p & 2180 & 2622 & 1247 & 903 & 1602 & 2148 \\
\hline miR-148b-3p & 1009 & 974 & 0 & 728 & 0 & 0 \\
\hline miR-149-5p & 1796 & 720 & 0 & 0 & 1292 & 0 \\
\hline miR-150-5p & 0 & 0 & 3595 & 3486 & 1920 & 1511 \\
\hline
\end{tabular}


Table 2. Cont.

\begin{tabular}{|c|c|c|c|c|c|c|}
\hline & \multicolumn{2}{|c|}{ MSC } & \multicolumn{2}{|c|}{ ssEV } & \multicolumn{2}{|c|}{ lsEV } \\
\hline & $-\mathrm{IFN} \gamma$ & $+\mathrm{IFN} \gamma$ & $-\mathrm{IFN} \gamma$ & $+\mathrm{IFN} \gamma$ & $-\mathrm{IFN} \gamma$ & $+\mathrm{IFN} \gamma$ \\
\hline miR-152-3p & 1854 & 2393 & 0 & 0 & 1209 & 1746 \\
\hline miR-15a-5p & 9268 & 11,252 & 8791 & 6577 & 10,976 & 7963 \\
\hline miR-15b-5p & 10,225 & 11,817 & 7676 & 4862 & 12,178 & 8735 \\
\hline miR-16-5p & 23,877 & 29,760 & 24,744 & 19,313 & 33,102 & 24,885 \\
\hline miR-17-5p & 3092 & 3465 & 3345 & 2828 & 3773 & 3677 \\
\hline miR-181a-3p & 270 & 0 & 0 & 0 & 0 & 0 \\
\hline miR-181a-5p & 2155 & 1557 & 1241 & 1021 & 1466 & 1414 \\
\hline miR-181b-5p & 944 & 634 & 0 & 0 & 0 & 0 \\
\hline miR-181d-5p & 714 & 473 & 0 & 0 & 0 & 0 \\
\hline miR-183-5p & 527 & 365 & 0 & 0 & 0 & 0 \\
\hline miR-185-5p & 610 & 540 & 0 & 632 & 0 & 0 \\
\hline miR-186-5p & 1164 & 1372 & 1963 & 1844 & 1696 & 1709 \\
\hline miR-18a-5p & 437 & 457 & 0 & 0 & 0 & 0 \\
\hline miR-191-5p & 3574 & 3677 & 3092 & 2965 & 3710 & 4040 \\
\hline miR-193a-3p & 3563 & 2401 & 0 & 587 & 2061 & 2227 \\
\hline miR-195-5p & 374 & 804 & 0 & 0 & 1055 & 0 \\
\hline miR-196a-5p & 5952 & 4730 & 1297 & 602 & 3301 & 3194 \\
\hline miR-196b-3p & 815 & 223 & 0 & 0 & 0 & 0 \\
\hline miR-196b-5p & 2520 & 2101 & 0 & 0 & 1290 & 0 \\
\hline miR-199a-5p & 15,996 & 17,854 & 6145 & 3584 & 13,123 & 13,706 \\
\hline miR-19a-3p & 1182 & 1504 & 2363 & 2298 & 2326 & 1862 \\
\hline miR-19b-3p & 6114 & 5510 & 6119 & 5431 & 6073 & 5334 \\
\hline miR-204-3p & 992 & 285 & 0 & 578 & 0 & 0 \\
\hline miR-205-5p & 0 & 117 & 0 & 0 & 1655 & 0 \\
\hline miR-20a-5p & 1715 & 2219 & 2308 & 1860 & 2061 & 2192 \\
\hline miR-20b-5p & 1504 & 1628 & 1924 & 1286 & 2043 & 1913 \\
\hline miR-210-3p & 7921 & 5347 & 1569 & 1288 & 3041 & 3329 \\
\hline miR-214-5p & 772 & 860 & 0 & 0 & 0 & 0 \\
\hline $\operatorname{miR}-21-5 p$ & 56,378 & 115,717 & 50,802 & 29,227 & 84,586 & 76,286 \\
\hline miR-218-5p & 1364 & 3301 & 0 & 0 & 942 & 1318 \\
\hline miR-221-3p & 60,280 & 36,507 & 17,469 & 9313 & 37,071 & 32,350 \\
\hline $\operatorname{miR}-222-3 p$ & 45,874 & 33,045 & 13,637 & 6941 & 31,186 & 26,064 \\
\hline miR-223-3p & 0 & 0 & 10,435 & 11,464 & 3899 & 3621 \\
\hline $\operatorname{miR}-22-3 p$ & 30,464 & 35,897 & 9711 & 5829 & 19,711 & 16,146 \\
\hline $\operatorname{miR}-22-5 p$ & 831 & 857 & 0 & 0 & 0 & 0 \\
\hline miR-23a-3p & 14,530 & 15,288 & 9451 & 6039 & 17,685 & 18,471 \\
\hline miR-23a-5p & 1364 & 318 & 0 & 0 & 1541 & 1513 \\
\hline miR-23b-3p & 9879 & 11,605 & 7103 & 4374 & 12,388 & 13,316 \\
\hline miR-24-3p & 14,019 & 13,811 & 5365 & 3360 & 10,476 & 10,575 \\
\hline $\operatorname{miR}-25-3 p$ & 3410 & 3478 & 4018 & 4686 & 3312 & 3240 \\
\hline
\end{tabular}


Table 2. Cont.

\begin{tabular}{|c|c|c|c|c|c|c|}
\hline & \multicolumn{2}{|c|}{ MSC } & \multicolumn{2}{|c|}{ ssEV } & \multicolumn{2}{|c|}{ lsEV } \\
\hline & $-\mathrm{IFN} \gamma$ & $+\mathrm{IFN} \gamma$ & $-\mathrm{IFN} \gamma$ & $+\mathrm{IFN} \gamma$ & $-\mathrm{IFN} \gamma$ & $+\mathrm{IFN} \gamma$ \\
\hline miR-26a-5p & 14,943 & 24,472 & 11,473 & 8941 & 16,360 & 14,933 \\
\hline miR-26b-5p & 4340 & 5251 & 5304 & 4160 & 5172 & 4732 \\
\hline miR-27a-3p & 9509 & 9645 & 11,580 & 5186 & 18,358 & 13,643 \\
\hline miR-27a-5p & 542 & 183 & 0 & 0 & 0 & 0 \\
\hline $\operatorname{miR}-27 b-3 p$ & 3745 & 5331 & 5673 & 2863 & 10,455 & 7796 \\
\hline $\operatorname{miR}-28-5 p$ & 2498 & 2798 & 0 & 0 & 1707 & 1785 \\
\hline miR-29a-3p & 32,286 & 36,765 & 14,087 & 7669 & 29,072 & 29,852 \\
\hline miR-29a-5p & 442 & 545 & 0 & 0 & 0 & 0 \\
\hline miR-29b-3p & 33,224 & 43,943 & 15,536 & 7714 & 27,775 & 26,096 \\
\hline $\operatorname{miR}-29 c-3 p$ & 1299 & 3281 & 1722 & 1594 & 3460 & 3008 \\
\hline miR-301a-3p & 1613 & 1329 & 1057 & 687 & 1350 & 1342 \\
\hline miR-30a-3p & 431 & 298 & 0 & 0 & 0 & 0 \\
\hline miR-30a-5p & 3415 & 4477 & 2124 & 1653 & 3523 & 3333 \\
\hline $\operatorname{miR}-30 b-5 p$ & 3544 & 4877 & 4024 & 3000 & 5261 & 4684 \\
\hline miR-30c-2-3p & 253 & 122 & 0 & 0 & 0 & 0 \\
\hline miR-30c-5p & 5151 & 7006 & 4198 & 2929 & 5608 & 4956 \\
\hline miR-30d-5p & 4961 & 5492 & 2658 & 1607 & 4626 & 4038 \\
\hline miR-30e-3p & 464 & 299 & 0 & 0 & 0 & 0 \\
\hline miR-30e-5p & 1188 & 1537 & 1222 & 953 & 1537 & 1351 \\
\hline miR-320a & 2177 & 1001 & 0 & 576 & 1707 & 1609 \\
\hline miR-324-5p & 970 & 620 & 0 & 0 & 0 & 0 \\
\hline miR-328-3p & 1955 & 975 & 0 & 1194 & 1092 & 1342 \\
\hline miR-331-3p & 829 & 642 & 0 & 0 & 0 & 0 \\
\hline miR-33a-5p & 2930 & 2128 & 0 & 625 & 1409 & 1453 \\
\hline miR-342-3p & 0 & 154 & 1680 & 1424 & 0 & 0 \\
\hline $\operatorname{miR}-34 a-5 p$ & 2393 & 2012 & 0 & 0 & 1242 & 1193 \\
\hline $\operatorname{miR}-34 c-5 p$ & 8039 & 7379 & 2217 & 1038 & 4598 & 3652 \\
\hline miR-361-5p & 304 & 478 & 0 & 0 & 0 & 0 \\
\hline miR-374b-5p & 685 & 749 & 0 & 0 & 1272 & 0 \\
\hline miR-425-5p & 1256 & 1249 & 1076 & 1122 & 966 & 0 \\
\hline miR-451a & 0 & 0 & 194,289 & 267,727 & 46,810 & 40,211 \\
\hline $\operatorname{miR}-484$ & 6203 & 5410 & 2543 & 1564 & 4918 & 5300 \\
\hline miR-486-5p & 0 & 146 & 3971 & 5677 & 1179 & 0 \\
\hline miR-532-3p & 354 & 254 & 1239 & 2490 & 0 & 1400 \\
\hline miR-532-5p & 251 & 258 & 0 & 0 & 0 & 0 \\
\hline miR-574-3p & 5199 & 3713 & 48,691 & 74,742 & 25,101 & 48,180 \\
\hline miR-574-5p & 20,670 & 18,789 & 180,234 & 217,257 & 78,303 & 149,525 \\
\hline miR-615-3p & 1621 & 944 & 0 & 0 & 1150 & 0 \\
\hline miR-652-3p & 1455 & 1110 & 0 & 860 & 927 & 0 \\
\hline miR-671-5p & 431 & 149 & 0 & 0 & 0 & 0 \\
\hline
\end{tabular}


Table 2. Cont.

\begin{tabular}{|c|c|c|c|c|c|c|}
\hline & \multicolumn{2}{|c|}{ MSC } & \multicolumn{2}{|c|}{ ssEV } & \multicolumn{2}{|c|}{ lsEV } \\
\hline & $-\mathrm{IFN} \gamma$ & $+\mathrm{IFN} \gamma$ & $-\mathrm{IFN} \gamma$ & $+\mathrm{IFN} \gamma$ & $-\mathbf{I F N} \gamma$ & $+\mathrm{IFN} \gamma$ \\
\hline miR-6766-3p & 3846 & 222 & 0 & 1818 & 0 & 0 \\
\hline miR-7-1-3p & 346 & 307 & 0 & 0 & 0 & 0 \\
\hline miR-744-5p & 254 & 141 & 0 & 0 & 0 & 0 \\
\hline miR-7-5p & 9101 & 10,666 & 3405 & 897 & 6088 & 3271 \\
\hline miR-877-5p & 284 & 0 & 0 & 0 & 0 & 0 \\
\hline miR-92b-3p & 1302 & 1503 & 0 & 866 & 1478 & 1527 \\
\hline miR-93-5p & 3329 & 3218 & 3205 & 2549 & 3023 & 3064 \\
\hline miR-96-5p & 1243 & 965 & 0 & 0 & 0 & 0 \\
\hline miR-99a-5p & 7734 & 8614 & 2852 & 1730 & 5380 & 5383 \\
\hline miR-99b-3p & 550 & 288 & 0 & 0 & 0 & 0 \\
\hline miR-99b-5p & 6388 & 5799 & 2252 & 1342 & 5311 & 4932 \\
\hline
\end{tabular}

A Upregulated miRNAs with IFN $\gamma$ treatment

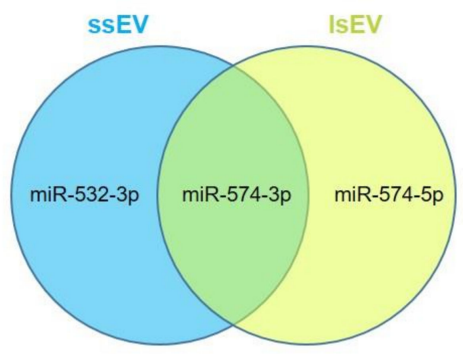

B

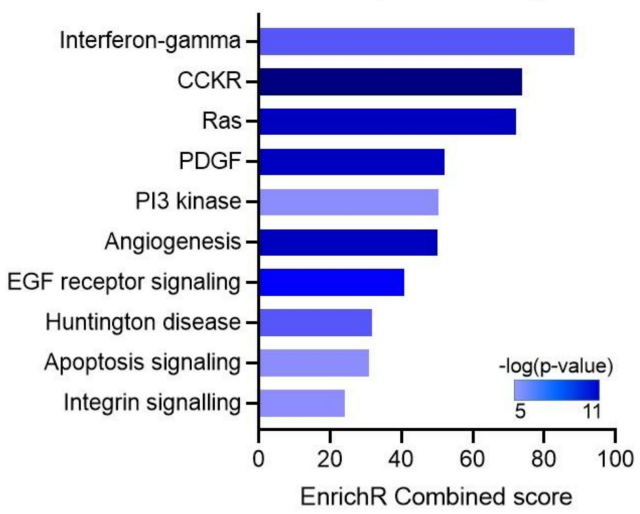

Downregulated miRNAs with IFN $\gamma$ treatment

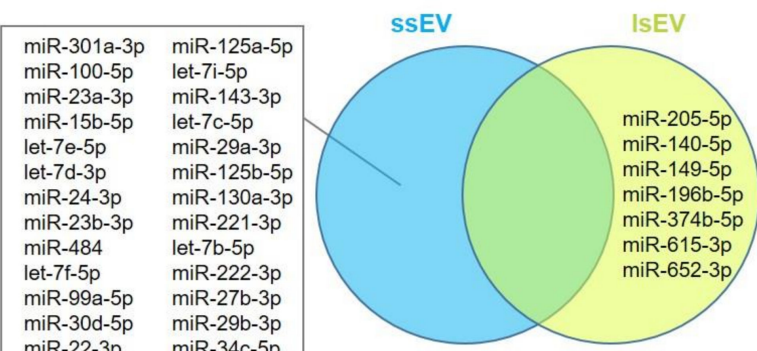

Ontologies

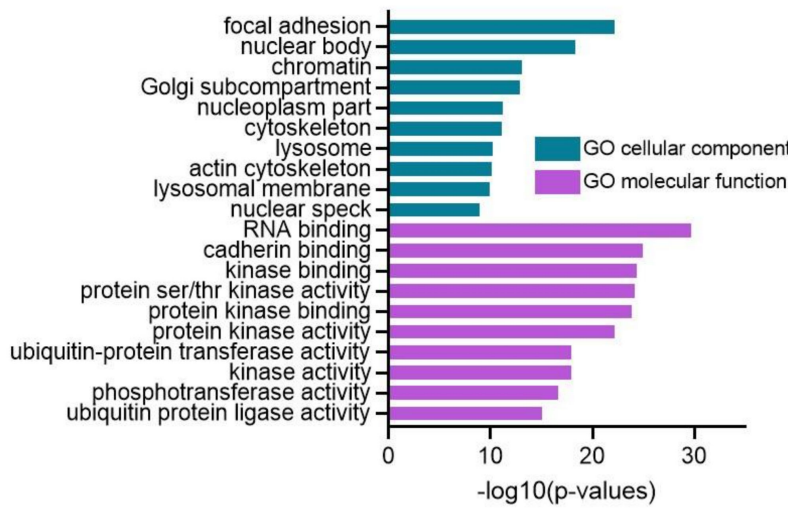

Figure 5. miRNAs modulated in MSC-EV subtypes after pre-activation with IFN $\gamma$. (A) Venn diagrams of the miRNAs that are up- and down-regulated in ssEVs A20 or lsEVs A20 (left and right panels, respectively) compared to ssEV NA or lsEV NA. (B) Gene Ontology enrichment analysis of biological pathways for the target genes of miRNAs modulated with IFN $\gamma$ from (A) with a fold change>1.5. (C) Gene Ontology enrichment analysis of biological pathways for cellular components and molecular functions of the target genes of miRNAs modulated with IFN $\gamma$ from (A).

We then quantified the expression of known immunosuppressive and anti-fibrotic markers in MSCs. At least one of the two doses of IFN $\gamma$ up-regulated Il1ra, Il6 and Cox2 mRNAs in MSCs while the expression of $i N o s, T s g 6, H g f$ and Tgf $\beta 1$ was not significantly modulated although $i$ Nos and Tsg 6 mRNA increased by a 20-fold and 2-fold factor, respectively (Figure 6A). 

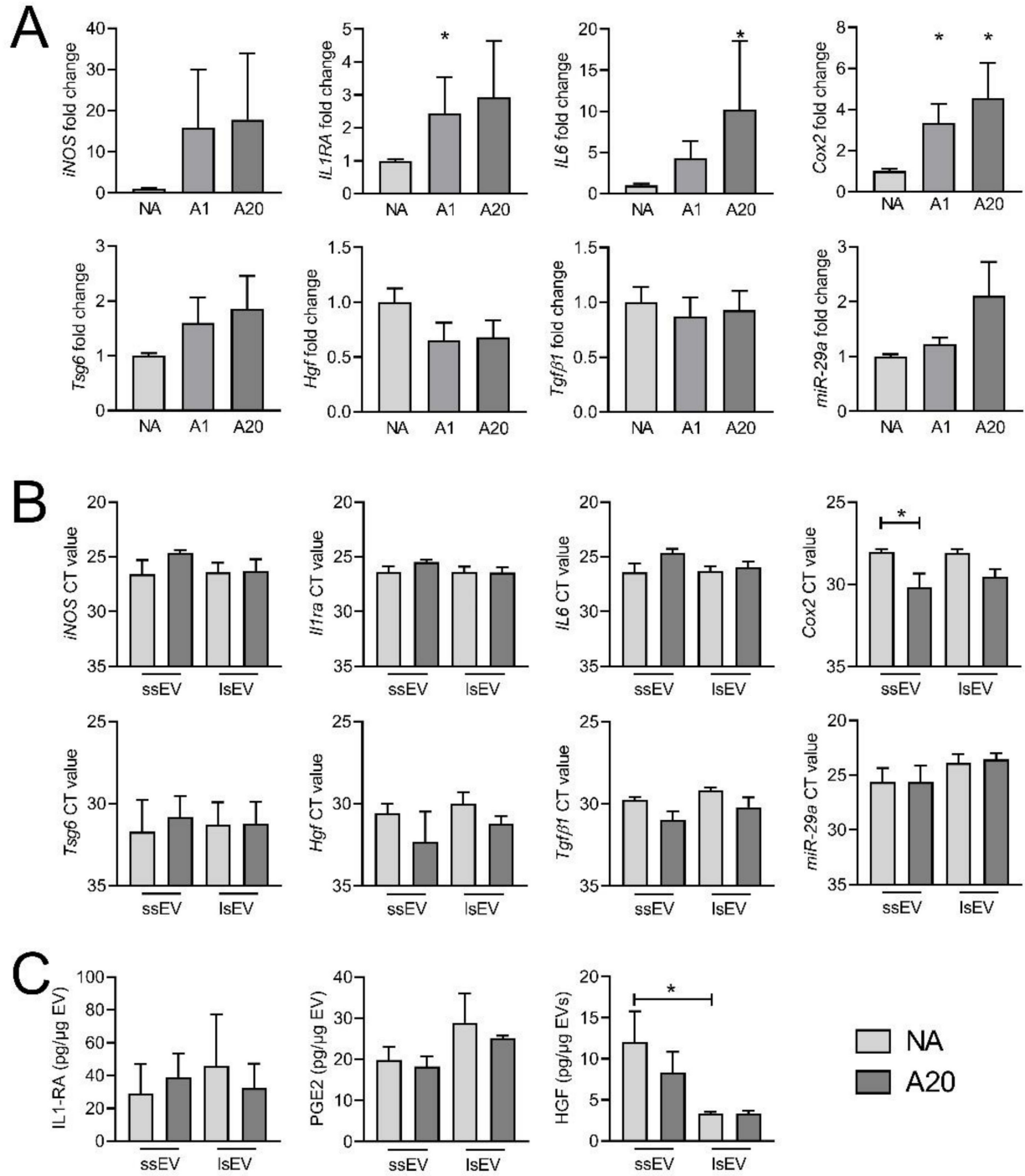

Figure 6. Immunosuppressive factors up-regulated in MSCs and in MSC-EVs after pre-activation with IFN $\gamma$. (A) Gene expression of known immunosuppressive and anti-fibrotic markers in non-activated MSCs (NA) or MSCs pre-activated with 1 (A1) or $20 \mathrm{ng} / \mathrm{mL}$ (A20) IFN $\gamma$. (B) Gene expression of known immunosuppressive and anti-fibrotic markers in ssEV NA, lsEV NA, ssEV A20 and lsEV A20). (C) Protein quantification of known immunosuppressive and anti-fibrotic markers in ssEV NA, lsEV NA, ssEV A20 and lsEV A20 obtained by ELISA. Data are presented as mean \pm SEM $(n=3)$; $*: p<0.05$ versus NA mMSCs or the indicated group control.

Expression of miR-29a-3p, which was shown to be involved in the beneficial effect of MSC-derived EVs in SSc [8], tended to be up-regulated in MSCs pre-activated by the high dose of IFN $\gamma$. Those markers were then quantified at the mRNA level in ssEV A20 and lsEV A20. No difference in the mRNA levels of the different markers was observed between ssEVs and lsEVs from non-activated and high dose IFN $\gamma$-pre-activated MSCs (Figure 6B). Nevertheless, a tendency to higher levels of iNos, Il1 ra and Il6 were observed in ssEV A20 compared to ssEV NA, suggesting that part of the mRNA from the parental cells were packaged in ssEVs. At the protein level, IL6 and TGF $\beta 1$ were not detected in any types of EVs while IL1-RA and PGE2 were slightly higher in lsEVs than ssEVs (Figure 6C). By contrast, HGF levels were lower in both non-activated or IFN $\gamma$ pre-activated lsEVs compared to ssEVs. In summary, IFN $\gamma$ pre-activation up-regulated the expression of several anti-inflammatory factors in mMSCs that were not found in EVs, suggesting that other factors might be related to the improvement of SSc markers in the lungs. 


\section{Discussion}

In the present study, we report that IFN $\gamma$ pre-activation of MSCs improved the therapeutic effect of EVs in the lungs of SSc-induced mice with no major impact on the skin.

We first showed that the therapeutic efficacy of MSC-EVs was dose-dependent. Interestingly, increasing the dose of lsEVs by a six-fold factor abolished the beneficial effect of EVs. This is consistent with our previous study that demonstrated that increasing the quantity of systemically injected MSCs from $2.5 \times 10^{5}$ cells to $1 \times 10^{6}$ cells reversed the beneficial role of cell therapy [6]. The reason for this is not known. This might be related to the local accumulation of EVs that might contribute to a dysregulated crosstalk with surrounding endogenous cells in a context of oxidative stress and inflammation related to SSc. This might also be related to the raise of a number of factors in serum, including inflammatory cytokines or profibrotic factors to levels that can induce unwanted effects on organs or immune cells and counteract the beneficial effects. The only markers that improved following infusion of the high lsEV dose were anti-oxidative stress genes in lungs. Whether this reflects an adaptive response to the elevation of oxidative stress in the lungs of mice receiving the high dose of lsEVs remains to be elucidated. Nevertheless, the data indicate that the optimal dose will have to be determined in larger preclinical models before clinical translation.

One important finding of the study is the interest in using EVs from IFN $\gamma$ pre-activated MSCs to enhance their beneficial effect in the lungs of SSc mice. It has already been shown that MSC priming by hypoxia or genetic modification may enhance their therapeutic properties in other applications $[19,20]$. Improvement of fibrotic, remodeling and inflammatory markers was observed by comparison with EVs from non-activated MSCs. Few studies have investigated the interest of IFN $\gamma$ pre-activation of MSCs before EV isolation. Nevertheless, EVs isolated from IFN $\gamma$ pre-activated human umbilical cord-derived MSCs were shown to increase the survival of rats with Escherichia coli-induced pneumonia and to reduce the lung injury [21]. EVs from IFN $\gamma$ pre-activated human BM-MSCs reduced experimental autoimmune encephalomyelitis (EAE) via the generation of Treg cells [22]. By contrast, EVs from primed human cord blood-derived MSCs were not able to protect kidney from ischemia-reperfusion injury or displayed similar efficacy as naïve EVs in experimental spinal cord injury $[23,24]$. In vitro, EVs isolated from human MSCs, whether pre-activated or not with IFN $\gamma$, exerted similar ability to inhibit T-cell proliferation although only preactivated EVs contained IDO mRNA [25] and a number of anti-inflammatory RNAs and proteins [22]. In addition to IDO and iNOS in human and mouse MSCs, respectively, up-regulation of PDL1 or CD200 was also proposed to sustain the enhanced immunosuppressive function of IFN $\gamma$ pre-activated MSC-EVs [9]. In our study, as expected, we found an up-regulation of several known immunosuppressive markers in IFN $\gamma$ pre-activated MSCs. The mRNAs of several of those markers tended to be up-regulated in ssEV A20 and might be related to the improvement of SSc-associated markers in the lungs. These markers were not up-regulated in $1 \mathrm{sEV} \mathrm{A20,} \mathrm{suggesting} \mathrm{that} \mathrm{they} \mathrm{are} \mathrm{differentially} \mathrm{routed}$ according to the biogenesis pathways of EVs. Furthermore, these results suggest that EVs can probably transfer mRNAs to target tissues where they can be translated into effector proteins regulating intrinsic pathways in the recipient cells. Interestingly, the mRNA levels of Cox2 were down-regulated in both ssEV A20 and lsEV A20 whereas the quantity of the protein PGE2 was not affected. Nevertheless, the quantity of PGE2 was higher in lsEVs compared to ssEVs, while HGF was lower. We did not found a higher content of miR-29a-3p in lsEVs, which is consistent with the limited effect of IFN $\gamma$ pre-activation on the miRNA landscape of human MSC-EVs [26]. The differential effect of EVs in the skin and lungs of SSc mice might be related to the route of administration and a higher effect in the lungs, which are primarily targeted using systemic administration. Another hypothesis is the up-regulation of specific soluble and membrane markers, which could impact their biodistribution and act differentially on different organs. To our knowledge, no differential effect of pre-activated versus naïve MSC-EVs has been described on organ functions so far and therefore a better understanding of IFN $\gamma$-pre-activation on EV functions is needed. 
Beside functional differences between EVs isolated from naïve and pre-activated MSCs, we also observed a slightly but constant higher therapeutic effect of lsEV A20 compared to ssEV A20 in the skin and lungs of treated mice. Controversial data are reported from the literature. SsEVs have been previously shown to outperform lsEVs in collagen-induced arthritis, kidney injury or in delayed-T hypersensitivity mouse models [27-29]. In another model, the proportion of ssEVs versus lsEVs was decreased after IFN $\gamma$ pre-activation and related to a concomitant loss of therapeutic efficiency, suggesting that lsEVs were more efficient than ssEVs [23]. Differences in protein, mRNA and miRNA content were proposed to explain the differential regenerative capacities of EV subtypes in a model of acute kidney injury [27]. The proproliferative effect of ssEVs was ascribed to the presence of many factors playing a role in the maintenance of cell cycle, while p53, a negative regulator of cell cycle, was found in lsEVs. Differences in membrane markers expressed by EV subtypes could also explain differential targeting of injured or diseased tissue since MSC-EVs rapidly localized in the injured organs and remained up to seven days after systemic administration [30]. The different cargo of ssEVs and lsEVs, and their inherent functions can differently impact the targeted tissues according to the disease. Further studies are therefore needed to decipher the respective roles of ssEVs and lsEVs, keeping in mind that the current isolation protocol does not allow for the isolation of pure populations of exosomes or microvesicles.

In conclusion, we showed that IFN $\gamma$ pre-activation of MSCs enhanced the beneficial effect of ssEVs and lsEVs by regulating several markers whose expression is altered in SSc. We further observed that IFN $\gamma$ pre-activated lsEVs might be more efficient than ssEVs in this specific disease application.

Supplementary Materials: The following are available online at https:/ /www.mdpi.com/article/10 $.3390 /$ cells10102727/s1, Figure S1: Dose effect of lsEVs isolated from MSCs in the murine model of HOCl-induced SSc.

Author Contributions: D.N., P.G., C.J. designed the experiments. Experimental work was performed by P.R., M.M., K.T. and data were analyzed by P.R., M.M., A.T.J.M.; P.R. and D.N. prepared the manuscript. All authors have contributed to revising the manuscript and final approval. All authors have read and agreed to the published version of the manuscript.

Funding: We gratefully acknowledge the Agence Nationale pour la Recherche for support of the national infrastructure: "ECELLFRANCE: Development of a national adult mesenchymal stem cell based therapy platform" (ANR-11-INSB-005). We also acknowledge the Réseau d'Histologie Expérimentale de Montpellier" histology facility for processing our tissues and, the "SMARTY platform and Network of Animal facilities of Montpellier".

Institutional Review Board Statement: The study was conducted according to the guidelines of the Declaration of Helsinki, and approved by the Regional Ethics Committee on Animal Experimentation (APAFIS\#53512016050919079187).

Informed Consent Statement: Not applicable.

Data Availability Statement: All data have been made available in the document.

Acknowledgments: We acknowledge funding support from the Inserm Institute, the University of Montpellier, the University Hospital of Montpellier, the Association des Sclérodermiques de France and the Société Nationale Française de Médecine Interne.

Conflicts of Interest: The authors disclose any financial or personal conflict of interest.

\section{References}

1. Denton, C.P.; Khanna, D. Systemic sclerosis. Lancet 2017, 390, 1685-1699. [CrossRef]

2. Tyndall, A. Hematopoietic Stem Cell Transplantation for Systemic Sclerosis: Review of Current Status. BioDrugs 2019, 33, 401-409. [CrossRef] [PubMed]

3. Rozier, P.; Maria, A.; Goulabchand, R.; Jorgensen, C.; Guilpain, P.; Noël, D. Mesenchymal Stem Cells in Systemic Sclerosis: Allogenic or Autologous Approaches for Therapeutic Use? Front. Immunol. 2018, 9, 2938. [CrossRef] [PubMed]

4. Van Niel, G.; D'Angelo, G.; Raposo, G. Shedding light on the cell biology of extracellular vesicles. Nat. Rev. Mol. Cell Biol. 2018, 19, 213-228. [CrossRef] [PubMed] 
5. Théry, C.; Amigorena, S.; Raposo, G.; Clayton, A. Isolation and Characterization of Exosomes from Cell Culture Supernatants and Biological Fluids. Curr. Protoc. Cell Biol. 2006, 30, 3.22.1-3.22.29. [CrossRef]

6. Maria, A.T.J.; Toupet, K.; Bony, C.; Pirot, N.; Vozenin, M.-C.; Petit, B.; Roger, P.; Batteux, F.; Le Quellec, A.; Jorgensen, C.; et al. Antifibrotic, Antioxidant, and Immunomodulatory Effects of Mesenchymal Stem Cells in HOCl-Induced Systemic Sclerosis. Arthritis Rheumatol. 2016, 68, 1013-1025. [CrossRef]

7. Maria, A.T.; Toupet, K.; Maumus, M.; Fonteneau, G.; Le Quellec, A.; Jorgensen, C.; Guilpain, P.; Noël, D. Human adipose mesenchymal stem cells as potent anti-fibrosis therapy for systemic sclerosis. J. Autoimmun. 2016, 70, 31-39. [CrossRef]

8. Rozier, P.; Maumus, M.; Maria, A.T.J.; Toupet, K.; Lai-Kee-Him, J.; Jorgensen, C.; Guilpain, P.; Noël, D. Mesenchymal stromal cells-derived extracellular vesicles alleviate systemic sclerosis via miR-29a-3p. J. Autoimmun. 2021, 121, 102660. [CrossRef]

9. Sivanathan, K.N.; Gronthos, S.; Rojas-Canales, D.; Thierry, B.; Coates, P.T. Interferon-Gamma Modification of Mesenchymal Stem Cells: Implications of Autologous and Allogeneic Mesenchymal Stem Cell Therapy in Allotransplantation. Stem Cell Rev. Rep. 2014, 10, 351-375. [CrossRef]

10. Szabó, E.; Fajka-Boja, R.; Kriston-Pál, E.; Hornung, A.; Makra, I.; Kudlik, G.; Uher, F.; Katona, R.L.; Monostori, E.; Czibula, A. Licensing by Inflammatory Cytokines Abolishes Heterogeneity of Immunosuppressive Function of Mesenchymal Stem Cell Population. Stem Cells Dev. 2015, 24, 2171-2180. [CrossRef]

11. Wobma, H.M.; Kanai, M.; Ma, S.P.; Shih, Y.; Li, H.W.; Duran-Struuck, R.; Winchester, R.; Goeta, S.; Brown, L.M.; Vunjak-Novakovic, G. Dual IFN- $\gamma /$ hypoxia priming enhances immunosuppression of mesenchymal stromal cells through regulatory proteins and metabolic mechanisms. J. Immunol. Regen. Med. 2018, 1, 45-56. [CrossRef]

12. Bouffi, C.; Bony, C.; Courties, G.; Jorgensen, C.; Noël, D. IL-6-Dependent PGE2 Secretion by Mesenchymal Stem Cells Inhibits Local Inflammation in Experimental Arthritis. PLoS ONE 2010, 5, e14247. [CrossRef] [PubMed]

13. Théry, C.; Witwer, K.W.; Aikawa, E.; Alcaraz, M.J.; Anderson, J.D.; Andriantsitohaina, R.; Antoniou, A.; Arab, T.; Archer, F.; Atkin-Smith, G.K.; et al. Minimal information for studies of extracellular vesicles 2018 (MISEV2018): A position statement of the International Society for Extracellular Vesicles and update of the MISEV2014 guidelines. J. Extracell. Vesicles 2018, 7, 1535750. [CrossRef]

14. Karagkouni, D.; Paraskevopoulou, M.D.; Chatzopoulos, S.; Vlachos, I.S.; Tastsoglou, S.; Kanellos, I.; Papadimitriou, D.; Kavakiotis, I.; Maniou, S.; Skoufos, G.; et al. DIANA-TarBase v8: A decade-long collection of experimentally supported miRNA-gene interactions. Nucleic Acids Res. 2017, 46, D239-D245. [CrossRef] [PubMed]

15. Kuleshov, M.V.; Jones, M.R.; Rouillard, A.D.; Fernandez, N.F.; Duan, Q.; Wang, Z.; Koplev, S.; Jenkins, S.L.; Jagodnik, K.M.; Lachmann, A.; et al. Enrichr: A comprehensive gene set enrichment analysis web server 2016 update. Nucleic Acids Res. 2016, 44, W90-W97. [CrossRef] [PubMed]

16. Xie, Z.; Bailey, A.; Kuleshov, M.V.; Clarke, D.J.B.; Evangelista, J.E.; Jenkins, S.L.; Lachmann, A.; Wojciechowicz, M.L.; Kropiwnicki, E.; Jagodnik, K.M.; et al. Gene Set Knowledge Discovery with Enrichr. Curr. Protoc. 2021, 1, e90. [CrossRef] [PubMed]

17. Chen, E.Y.; Tan, C.M.; Kou, Y.; Duan, Q.; Wang, Z.; Meirelles, G.V.; Clark, N.R.; Ma'Ayan, A. Enrichr: Interactive and collaborative HTML5 gene list enrichment analysis tool. BMC Bioinform. 2013, 14, 128. [CrossRef] [PubMed]

18. Ghannam, S.; Bouffi, C.; Djouad, F.; Jorgensen, C.; Noël, D. Immunosuppression by mesenchymal stem cells: Mechanisms and clinical applications. Stem Cell Res. Ther. 2010, 1, 1-7. [CrossRef]

19. Gregorius, J.; Wang, C.; Stambouli, O.; Hussner, T.; Qi, Y.; Tertel, T.; Börger, V.; Yusuf, A.M.; Hagemann, N.; Yin, D.; et al. Small extracellular vesicles obtained from hypoxic mesenchymal stromal cells have unique characteristics that promote cerebral angiogenesis, brain remodeling and neurological recovery after focal cerebral ischemia in mice. Basic Res. Cardiol. 2021, 116, 1-19. [CrossRef]

20. Paudyal, A.; Ghinea, F.S.; Driga, M.P.; Fang, W.-H.; Alessandri, G.; Combes, L.; Degens, H.; Slevin, M.; Hermann, D.M.; PopaWagner, A. p5 Peptide-Loaded Human Adipose-Derived Mesenchymal Stem Cells Promote Neurological Recovery After Focal Cerebral Ischemia in a Rat Model. Transl. Stroke Res. 2020, 12, 125-135. [CrossRef]

21. Varkouhi, A.K.; Jerkic, M.; Ormesher, L.; Gagnon, S.; Goyal, S.; Rabani, R.; Masterson, C.; Spring, C.; Chen, P.Z.; Gu, F.X.; et al. Extracellular Vesicles from Interferon- $\gamma$-primed Human Umbilical Cord Mesenchymal Stromal Cells Reduce Escherichia coli-induced Acute Lung Injury in Rats. Anesthesiology 2019, 130, 778-790. [CrossRef]

22. Riazifar, M.; Mohammadi, M.R.; Pone, E.J.; Yeri, A.; Lässer, C.; Segaliny, A.I.; McIntyre, L.L.; Shelke, G.; Hutchins, E.; Hamamoto, A.; et al. Stem Cell-Derived Exosomes as Nanotherapeutics for Autoimmune and Neurodegenerative Disorders. ACS Nano 2019, 13, 6670-6688. [CrossRef]

23. Kilpinen, L.; Impola, U.; Sankkila, L.; Ritamo, I.; Aatonen, M.; Kilpinen, S.; Tuimala, J.; Valmu, L.; Levijoki, J.; Finckenberg, P.; et al. Extracellular membrane vesicles from umbilical cord blood-derived MSC protect against ischemic acute kidney injury, a feature that is lost after inflammatory conditioning. J. Extracell. Vesicles 2013, 2, 21927. [CrossRef]

24. Ruppert, K.A.; Nguyen, T.T.; Prabhakara, K.S.; Furman, N.E.T.; Srivastava, A.; Harting, M.; Cox, C.S., Jr.; Olson, S.D. Human Mesenchymal Stromal Cell-Derived Extracellular Vesicles Modify Microglial Response and Improve Clinical Outcomes in Experimental Spinal Cord Injury. Sci. Rep. 2018, 8, 1-12. [CrossRef]

25. Serejo, T.R.T.; Silva-Carvalho, A.; Braga, L.D.D.C.F.; Neves, F.D.A.R.; Pereira, R.W.; De Carvalho, J.L.; Saldanha-Araujo, F. Assessment of the Immunosuppressive Potential of INF- $\gamma$ Licensed Adipose Mesenchymal Stem Cells, Their Secretome and Extracellular Vesicles. Cells 2019, 8, 22. [CrossRef] 
26. Peltzer, J.; Lund, K.; Goriot, M.-E.; Grosbot, M.; Lataillade, J.-J.; Mauduit, P.; Banzet, S. Interferon- $\gamma$ and Hypoxia Priming Have Limited Effect on the miRNA Landscape of Human Mesenchymal Stromal Cells-Derived Extracellular Vesicles. Front. Cell Dev. Biol. 2020, 8, 1343. [CrossRef] [PubMed]

27. Bruno, S.; Tapparo, M.; Collino, F.; Chiabotto, G.; Deregibus, M.C.; Lindoso, R.S.; Neri, F.; Kholia, S.; Giunti, S.; Wen, S.; et al. Renal Regenerative Potential of Different Extracellular Vesicle Populations Derived from Bone Marrow Mesenchymal Stromal Cells. Tissue Eng. Part A 2017, 23, 1262-1273. [CrossRef] [PubMed]

28. Cosenza, S.; Toupet, K.; Maumus, M.; Crawford, P.L.; Blanc-Brude, O.; Jorgensen, C.; Noël, D. Mesenchymal stem cells-derived exosomes are more immunosuppressive than microparticles in inflammatory arthritis. Theranostics 2018, 8, 1399-1410. [CrossRef] [PubMed]

29. Bruno, S.; Grange, C.; Collino, F.; Deregibus, M.C.; Cantaluppi, V.; Biancone, L.; Tetta, C.; Camussi, G. Microvesicles Derived from Mesenchymal Stem Cells Enhance Survival in a Lethal Model of Acute Kidney Injury. PLoS ONE 2012, 7, e33115. [CrossRef] [PubMed]

30. Varderidou-Minasian, S.; Lorenowicz, M.J. Mesenchymal stromal/stem cell-derived extracellular vesicles in tissue repair: Challenges and opportunities. Theranostics 2020, 10, 5979-5997. [CrossRef] [PubMed] 